\title{
DIFFERENTIAL RESPONSES IN DROSOPHILA MELANOGASTER TO ENVIRONMENTAL ETHANOL: MODIFICATION OF FITNESS COMPONENTS AT THE Adh LOCUS
}

\author{
G. DORADO AND M. BARBANCHO \\ Departamento de Genética, Facultad de Ciencias, Universidad \\ de Córdoba (Córdoba), Spain
}

Received 10.xi.83

\section{SUMMARY}

\begin{abstract}
Homozygous $A d h^{\mathrm{F}} A d h^{\mathrm{F}}$ and $A d h^{\mathrm{S}} A d h^{\mathrm{S}}$ lines, collected from the same $D$. melanogaster winery polymorphic population, were submitted to stress by environmental ethanol. The responses of each of the FF, FS and SS genotypes, for both control and selected lines were analysed in normal and ethanol supplemented medium, in relation to three fitness components: egg-to-adult viability, developmental time and ethanol utilisation. Ethanol selection has resulted in a higher ethanol tolerance, a shorter developmental time and a better ability to use ethanol as food, showing the $A d h^{\mathrm{F}} A d h^{\mathrm{F}}$ genotype to be that with the highest relative fitness.
\end{abstract}

\section{INTRODUCTION}

One of the most common ways to show that selection acts on a determined locus is to grow individuals in a medium supplemented with a specific substrate of the products of the locus under consideration. In this way, most of the studies done with $D$. melanogaster involve the Adh locus.

When polymorphic populations are grown in media supplemented with different alcohols, a considerable $A d h^{\mathrm{F}}$ increase has been observed (Gibson, 1970; Bijlsma-Meeles and van Delden, 1974; van Delden et al., 1975, 1978; Cavener and Clegg, 1978, 1981). On the other hand, differential responses of the Adh genotypes have been obtained in ethanol supplemented media for ethanol tolerance (Morgan, 1975; Briscoe et al., 1975; Oakeshott, 1976; van Delden et al., 1978; McKechnie and Morgan, 1982), or for the ability to use ethanol as food (Libion-Mannaert et al., 1976; David et al., 1976, 1981; Daly and Clarke, 1981). In general, in such media $A d h^{\mathrm{F}} \operatorname{Adh}^{\mathrm{F}}$ genotypes show greater fitness, such superiority being associated with a more active ADH-F isozyme (Gibson and Miklovich, 1971; LibionMannaert et al., 1976; Kamping and van Delden, 1978). However, there is evidence that ethanol tolerance and ethanol utilisation both seem to be, at least partially, controlled by different genetic mechanisms (van Herrewege and David, 1980; David et al., 1981). Besides, an increase for the ethanol tolerance is not invariably associated with an increase for $A d h^{\mathrm{F}}$ frequencies or with an ADH activity increase (Gibson et al., 1979; Oakeshott, 1979; Ziolo and Parsons, 1982).

But, apart from the above, there are other fitness components that may be important for the maintenance of the genetic variability at the $A d h$ locus. In fact, small differences in the developmental times can be an important component of variation of the fitness in species that, like $D$. melanogaster, 
feed constantly (Lewontin, 1974). Differences in the developmental times have been found between $A d h$ genotypes both on regular food (van Delden and Kamping, 1979) and on alcohol supplemented media (Oakeshott, 1976, 1977), the $A d h^{\mathrm{F}} A d h^{\mathrm{F}}$ homozygotes having developmental times shorter than the $A d h^{\mathrm{S}} A d h^{\mathrm{S}}$ ones. These differences in the developmental times seem to be linearly related to the differences in the ADH activity (Oakeshott, 1976).

All the above information suggests that $A d h$ variants are not neutral on regular laboratory food (see also Wilson et al., 1982) nor on alcoholic supplemented media (see also Clarke, 1975; Thompson and Kaiser, 1977; Cavener and Clegg, 1981). The direction of the selective differences does not always seem to be the same for the different components analysed.

In this paper, the responses of both the $A d h^{\mathrm{F}}$ and the $A d h^{\mathrm{S}}$ homozygous lines (and the hybrid one) to a selection imposed by environmental ethanol are studied with respect to egg-to-adult viability, developmental time and ethanol utilisation as food.

\section{MATERIALS AND METHODS}

The flies used came from a sample collected in the winery named "Lagar de los Reyes" (LR) in Baena, Córdoba (Spain). At the time that the flies were collected, LR population was polymorphic at the Adh locus with frequencies of 0.875 and 0.125 for the $A d h^{\mathrm{F}}$ and $A d h^{\mathrm{S}}$ alleles respectively. From the progeny of this sample, 15 homozygous $A d h^{\mathrm{F}}$ and 15 homozygous $A d h^{\mathrm{s}}$ stocks (each coming from one single and independent homozygote $\times$ homozygote cross) were founded. 10 males and 10 females of each homozygous stock ( 300 flies for each allele) were used to originate the $A d h^{\mathrm{F}}$ and $A d h^{\mathrm{S}}$ homozygous control lines (LRCF and LRCS). These lines were independently grown on normal medium for 10 generations before starting selection.

Selected $A d h^{\mathrm{F}}$ and $A d h^{\mathrm{S}}$ homozygous lines (LRSeF and LRSeS) came from each respective LRC line growing on ethanol-supplemented medium. The number of generations of selection was 16 . In order to maintain a high number of flies in the lines, two sub-lines of each were maintained and sib-mated each generation. To obtain heterozygous $A d h^{\mathrm{F}} A d h^{\mathrm{s}}$ (FS) flies, virgin $A d h^{\mathrm{F}} A d h^{\mathrm{F}}$ (FF) females and $A d h^{\mathrm{S}} A d h^{\mathrm{S}}$ (SS) males were mated.

Normal medium consists of $1000 \mathrm{ml}$ water, $100 \mathrm{~g}$ sucrose, $100 \mathrm{~g}$ fresh dead yeast, $12 \mathrm{~g}$ agar $0.5 \mathrm{~g}$ salt and $5 \mathrm{ml}$ propionic acid. An ethanolsupplemented medium was obtained by adding $11 \mathrm{ml}$ ethanol $(99.5$ per cent) to $89 \mathrm{ml}$ of normal medium with vigorous stirring after the temperature was below $50^{\circ} \mathrm{C}$.

To study egg-to-adult viability and developmental time, eggs laid by three-day-old females of the appropriate genotype were used; 200 per 1 litre bottle of normal medium and 800 per ethanol-supplemented medium. Adults were removed daily as they emerged.

In order to determine the relative abilities to use ethanol as food, 40 three day old flies ( 20 males and 20 females) were kept in $270 \mathrm{ml}$ glass vials containing $25 \mathrm{ml}$ of basic medium (12 per cent w/v agar in water) or ethanol-basic medium ( 11 per cent $v / v$ ethanol in basic medium). The $\operatorname{Ld}_{50}$ (day on which 50 per cent of the flies died) was used to evaluate ethanol utilisation. 
Both the maintenance of lines and the experiments were carried out at approximately $25^{\circ} \mathrm{C}$. In the experiments, two replicates for each genotype, medium and line were made and their mean values were obtained for the study of the different effects.

\section{Results}

Results for each line, genotype and medium for each of the fitness components studied are summarised in fig. 1. Numerical data for viability (as survival egg-to-adult percentages), developmental times (as days from egg-to-adult) and life span (as lethal days 50) are given in table 2.
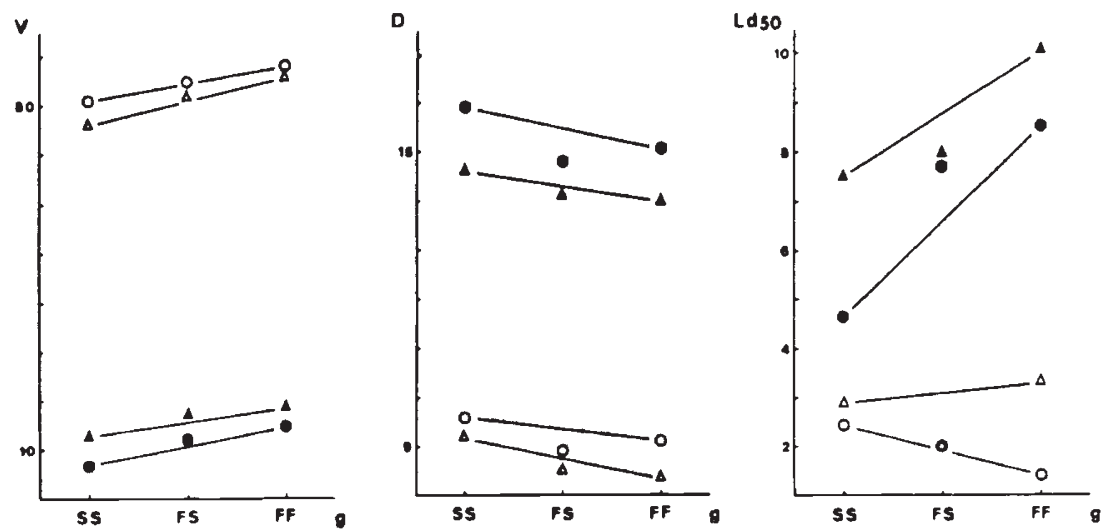

FIG. 1. Percentage survival into adults from eggs (V), mean developmental time, in days (D) and life span as lethal day 50 (Ld50) for each line, genotype and medium. Normal or basic medium: LRC $(O)$, LRSe $(\triangle)$. Supplemented medium: LRC $(\mathbf{O})$, LRSe $(\Delta)$.

TABLE 1

Factorial analysis of variance of the effects of line, genotype and medium on the egg-to-adult viability, developmental time and life duration

\begin{tabular}{|c|c|c|c|c|c|c|c|}
\hline \multirow[b]{2}{*}{$\begin{array}{l}\text { Source of } \\
\text { variation }\end{array}$} & \multirow[b]{2}{*}{ d.f. } & \multicolumn{2}{|c|}{ Viability } & \multicolumn{2}{|c|}{$\begin{array}{c}\text { Developmental } \\
\text { Time }\end{array}$} & \multicolumn{2}{|c|}{ Life span } \\
\hline & & $\begin{array}{l}\text { Mean } \\
\text { square }\end{array}$ & $\mathrm{F}$ & Desviance & $\mathrm{F}$ & $\begin{array}{l}\text { Mean } \\
\text { square }\end{array}$ & $F$ \\
\hline Lines (L) & 1 & 1.835 & $0.308 \mathrm{~ns}$ & $4 \cdot 002$ & $174 \cdot 175^{* *}$ & $8 \cdot 158$ & $203.871^{* *}$ \\
\hline Genotypes (G) & 2 & $116 \cdot 386$ & $19 \cdot 569^{* *}$ & $1 \cdot 203$ & $52 \cdot 376^{* *}$ & 4.469 & $111 \cdot 666^{* *}$ \\
\hline Mediums (M) & 1 & $28766 \cdot 450$ & $4836 \cdot 892^{* *}$ & $198 \cdot 720$ & $8649 \cdot 408^{* *}$ & $173 \cdot 887$ & $4345 \cdot 225^{* *}$ \\
\hline $\mathrm{L} \times \mathrm{G}$ & 2 & $0 \cdot 164$ & $0.028 \mathrm{~ns}$ & 0.125 & $5 \cdot 458^{*}$ & $1 \cdot 550$ & $38 \cdot 728^{* *}$ \\
\hline $\mathbf{L} \times \mathbf{M}$ & 1 & $106 \cdot 851$ & $17 \cdot 967^{* *}$ & 0.459 & $19.990^{* *}$ & 0.986 & $24 \cdot 644^{* *}$ \\
\hline $\mathrm{G} \times \mathrm{M}$ & 2 & 1.850 & $0.311 \mathrm{~ns}$ & 0.001 & $0.064 \mathrm{~ns}$ & $6 \cdot 642$ & $165 \cdot 968^{* *}$ \\
\hline $\mathrm{L} \times \mathrm{G} \times \mathbf{M}$ & 2 & $3 \cdot 717$ & $0.625 \mathrm{~ns}$ & $0 \cdot 105$ & $4.554^{*}$ & $1 \cdot 088$ & $27 \cdot 184^{* *}$ \\
\hline Residual & 12 & $5 \cdot 947$ & & 0.023 & & 0.040 & \\
\hline Total & 23 & & & & & & \\
\hline
\end{tabular}

$* \mathrm{P}<0.05,{ }^{* *} \mathrm{P}<0.01$.

\section{(i) ANOVA}

A factorial ANOVA (summarised in table 1) was used to test the extent of effect upon lines, genotypes and media for each fitness component 
TABLE 2

Mean values for each fitness component, genotype, line and medium. Genotype means not underlined by a common line are significantly different, at least at $P<0.05$

\begin{tabular}{|c|c|c|c|c|c|c|}
\hline \multirow{3}{*}{$\begin{array}{c}\text { Fitness } \\
\text { Component }\end{array}$} & \multicolumn{6}{|c|}{ Control lines } \\
\hline & \multicolumn{3}{|c|}{ Regular medium } & \multicolumn{3}{|c|}{ Supplemented medium } \\
\hline & SS & FS & FF & SS & FS & FF \\
\hline Viability & $\underline{81 \cdot 00}$ & $84 \cdot 75$ & 88.00 & 6.69 & $11 \cdot 94$ & $14 \cdot 75$ \\
\hline Developmental times & $9 \cdot 60$ & 8.90 & $9 \cdot 10$ & $15 \cdot 89$ & $14 \cdot 79$ & $15 \cdot 04$ \\
\hline Starvation resistance & $2 \cdot 44$ & 1.99 & $1 \cdot 41$ & & & \\
\hline Ethanol utilization & & & & $4 \cdot 64$ & $7 \cdot 68$ & $8 \cdot 50$ \\
\hline
\end{tabular}

\begin{tabular}{|c|c|c|c|c|c|c|}
\hline \multirow{3}{*}{$\begin{array}{c}\text { Fitness } \\
\text { Component }\end{array}$} & \multicolumn{6}{|c|}{ Selected lines } \\
\hline & \multicolumn{3}{|c|}{ Regular medium } & \multicolumn{3}{|c|}{ Supplemented medium } \\
\hline & SS & FS & FF & SS & FS & FF \\
\hline Viability & $75 \cdot 75$ & $81 \cdot 50$ & $85 \cdot 50$ & $12 \cdot 69$ & 16.94 & 18.06 \\
\hline Developmental times & $9 \cdot 20$ & 8.50 & $8 \cdot 35$ & $14 \cdot 60$ & $14 \cdot 13$ & 13.97 \\
\hline Starvation resistance & 2.89 & 1.94 & $3 \cdot 30$ & & & \\
\hline Ethanol utilization & & & & $7 \cdot 50$ & $7 \cdot 97$ & $10 \cdot 04$ \\
\hline
\end{tabular}

studied. Significant differences between lines, for life span and developmental times, indicate that the LRSe line has a higher life span and a shorter developmental time for both media than the LRC. No significant difference was observed for the ethanol tolerance component. However, when an ANOVA for each medium was done, significant differences for the ethanol tolerance in the supplemented medium and for life span and developmental times in both normal and supplemented media were observed.

Significant differences between genotypes were found for all three components, such differences depending on the line and medium (see table 2). These inter-genotypic differences were also found when an ANOVA for each particular medium was done. Significant differences between media were also found for all the components; ethanol produces negative effects on viability and developmental time, and positive ones at life span increases.

$\mathrm{L} \times \mathrm{G}$ interactions were significant for developmental times (interactions only being significant in the supplemented medium) and for life span (probably due to the different pattern of dominance in the supplemented medium).

The significant interactions $\mathrm{L} \times \mathrm{M}$ for all the components are related to a better response of the LRSe line in the supplemented medium (see fig. 1 and table 2).

Significant differences for $G \times M$ interactions have only been found for life span components and must be related to the highest resistance to starvation of the homozygous SS and the highest ethanol utilisation of the homozygous FF. As this component really includes two, its effects can be better analysed through an ANOVA for each particular medium. However, when this was done, similar significant differences were observed. 


\section{(ii) Genetic effects}

Intergenotypic differences for each line and medium were tested in table 2 by means of a student's test, by using the error mean squares of the previous analyses of variance. As the differences between genotypes were analysed within lines and within media, the error mean squares used were those of the analyses of variances done for each particular medium.

On the supplemented medium, for both lines, FF and FS individuals show egg-to-adult viabilities higher than that of the SS homozygotes. On the regular medium, though, dominance of the $A d h^{F}$ allele has not been observed, no significant differences being found among the genotypes of the control lines. On the other hand, small but significant differences can be observed for the developmental times. Apparently there is complete dominance of the $A d h^{\mathrm{F}}$ allele over the $A d h^{\mathrm{S}}$ one in this respect in both lines and media.

In relation to life span, the ability to use the ethanol as food is evaluated by the use of the ethanol-basic medium. Adult FF homozygotes are the best and adult SS homozygotes the worst at using ethanol for metabolic energy, the $A d h^{\text {s }}$ allele showing a dominant effect at the selected lines, though. By using basic medium, the resistance to starvation (basic medium only provides water to the flies) is evaluated, this being very different for the control and selected lines.

\section{(iii) Ethanol effects}

Ethanol effects for each genotype separately, for both control and selected lines, were quantified by the differences between data from supplemented and normal medium (see table 3 ). Differences are also tested in table 3 (normal vs. supplemented medium) by means of a student's test by using the error mean squares of the previous analyses of variances.

Highly significant differences $(p<0.001)$ are found for both control and selected lines for all three fitness components studied. For both LRC and

TABLE 3

Ethanol and selection effect and their statistical significance for each Adh genotype. Life span on normal medium (implying resistance to starvation) and on supplemented medium (ethanol utilisation). (+): loss of viability, delay in developmental time and life duration increase. (-): the opposite

\begin{tabular}{|c|c|c|c|c|c|}
\hline \multirow[b]{2}{*}{ Adh } & \multicolumn{2}{|c|}{ Ethanol effect } & \multicolumn{2}{|c|}{ Selection effect } & \\
\hline & $\begin{array}{c}\mathrm{LRC} \\
\mathrm{C}^{\mathrm{Su}}-\mathrm{C}^{\mathrm{N}}\end{array}$ & $\begin{array}{c}\text { LRSe } \\
\mathrm{Se}^{\mathrm{Su}}-\mathrm{Se}^{\mathrm{N}}\end{array}$ & $\underset{S e^{N}-C^{N}}{\stackrel{N}{N} \cdot}$ & $\begin{array}{l}\text { Su.M. } \\
\mathrm{Se}^{\mathrm{Su}}-\mathrm{C}^{\mathrm{Su}}\end{array}$ & \\
\hline SS & $+91.75^{* * *}$ & $+83 \cdot 30^{* * *}$ & $+6.17 \mathrm{~ns}$ & $-7 \cdot 42^{* * *}$ & \\
\hline FS & $+85.91^{* * * *}$ & $+79 \cdot 21^{* * *}$ & $+3.83 \mathrm{~ns}$ & $-5 \cdot 90^{* * *}$ & Viability \\
\hline FF & $+83 \cdot 24^{* * *}$ & $+79 \cdot 30^{* * *}$ & $+2.84 \mathrm{~ns}$ & $-3 \cdot 76^{* *}$ & \\
\hline SS & $+6.29^{* * *}$ & $+5 \cdot 40^{* * *}$ & $-0 \cdot 40^{*}$ & $-1 \cdot 29^{* * *}$ & \\
\hline FS & $+5.89^{* * *}$ & $+5.63^{* * *}$ & $-0 \cdot 40^{*}$ & $-0.66^{* *}$ & Developmental \\
\hline FF & $+5.94^{* * *}$ & $+5 \cdot 62^{* * *}$ & $-0.75^{* *}$ & $-1 \cdot 07^{* * *}$ & Time \\
\hline SS & $+2 \cdot 20^{* * *}$ & $+4 \cdot 61^{* * *}$ & $+0.45^{* *}$ & $+2 \cdot 86^{* * *}$ & \\
\hline FS & $+5 \cdot 70^{* * *}$ & $+6.03^{* * *}$ & $-0.05 \mathrm{~ns}$ & $+0.28 \mathrm{~ns}$ & Life \\
\hline FF & $+7.09^{* * * *}$ & $+6 \cdot 73^{* * *}$ & $+1.90^{* * *}$ & $+1 \cdot 54^{* *}$ & \\
\hline
\end{tabular}


LRSe lines, the ethanol provokes a great mortality increase (up to $91 \cdot 75$ per cent in LRC-S), a great delay in the developmental times (between 5 and 6 days) and a considerable life span increase when ethanol is the only food (until 7 days for LRC-F). However, negative (egg-to-adult viability and developmental time) effects are slighter and the positive effect (life span) more important for the selected lines with the exception of the $A d h^{\mathrm{F}}$ $A d h^{\mathrm{F}}$ genotype for life span (see also figs. 1, 2 and 3). This smaller life span increase in LRSe-F than in LRC-F may be understood by the exceptional starvation resistance increase provided by selection of the LRC-F.

\section{(iv) Selection effects}

Differences for significance are tested in table 3 by means of a student's test for each genotype separately for both normal and supplemented medium (LRC vs. LRSe lines). For this, in each respective case, the error mean squares used were that of analyses of variances separately done for each medium. In the absence of ethanol no changes in the egg-adult mortality were noted by the ethanol selection. However, in such normal medium, changes have been found in the developmental times for all three genotypes and in the life span for both homozygous ones. In the presence of ethanol, the same changes were brought about by the ethanol selection but, moreover, the ethanol tolerance of all three genotypes has been improved.

The effects of selection, quantified by the differences between data from selected and control lines, are also shown in table 3. By considering only the significative statistical values for both media, the ethanol selection produces an increase in the egg-to-adult viability (up to a 7.42 per cent of lower mortality), shorter developmental time (to 1.29 days in LRSe in

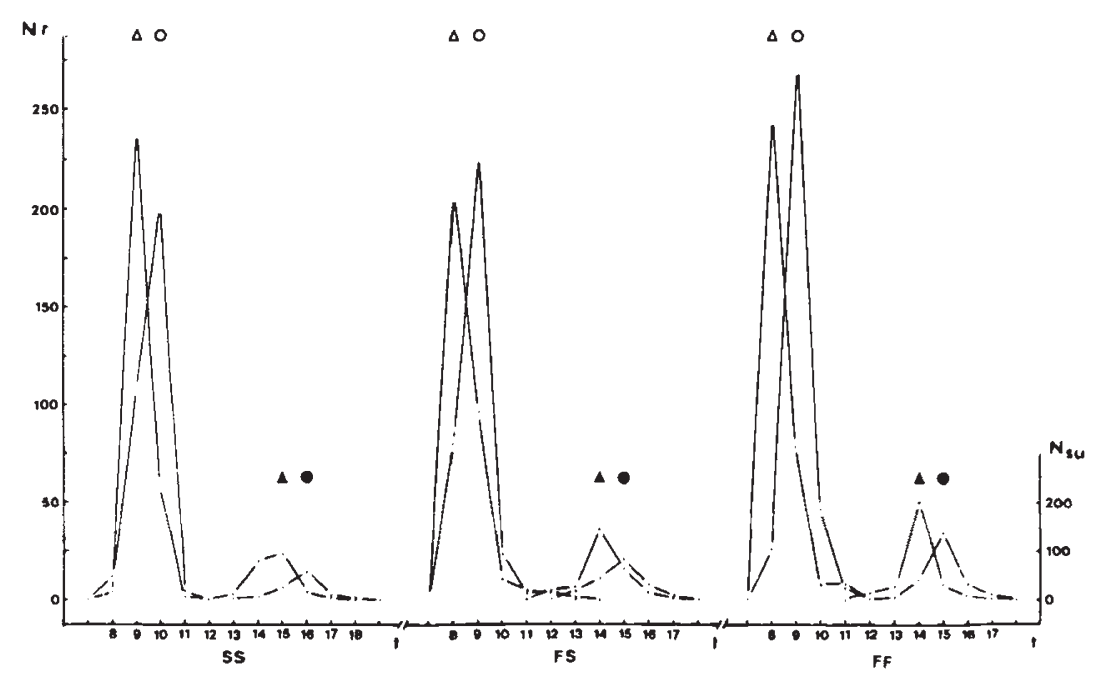

Fig. 2. Egg-to-adult developmental time-viability curves of LRC $(O, O)$ and LRSe $(\triangle, \Delta)$ on regular $(O, \triangle)$ and supplemented $(O, \Delta)$ mediums for each $A d h$ genotype, as a function of the number of days (t). 400 eggs on regular medium $(\mathrm{Nr})$ and 1600 on the supplemented one (Nsu) were used for each curve. 


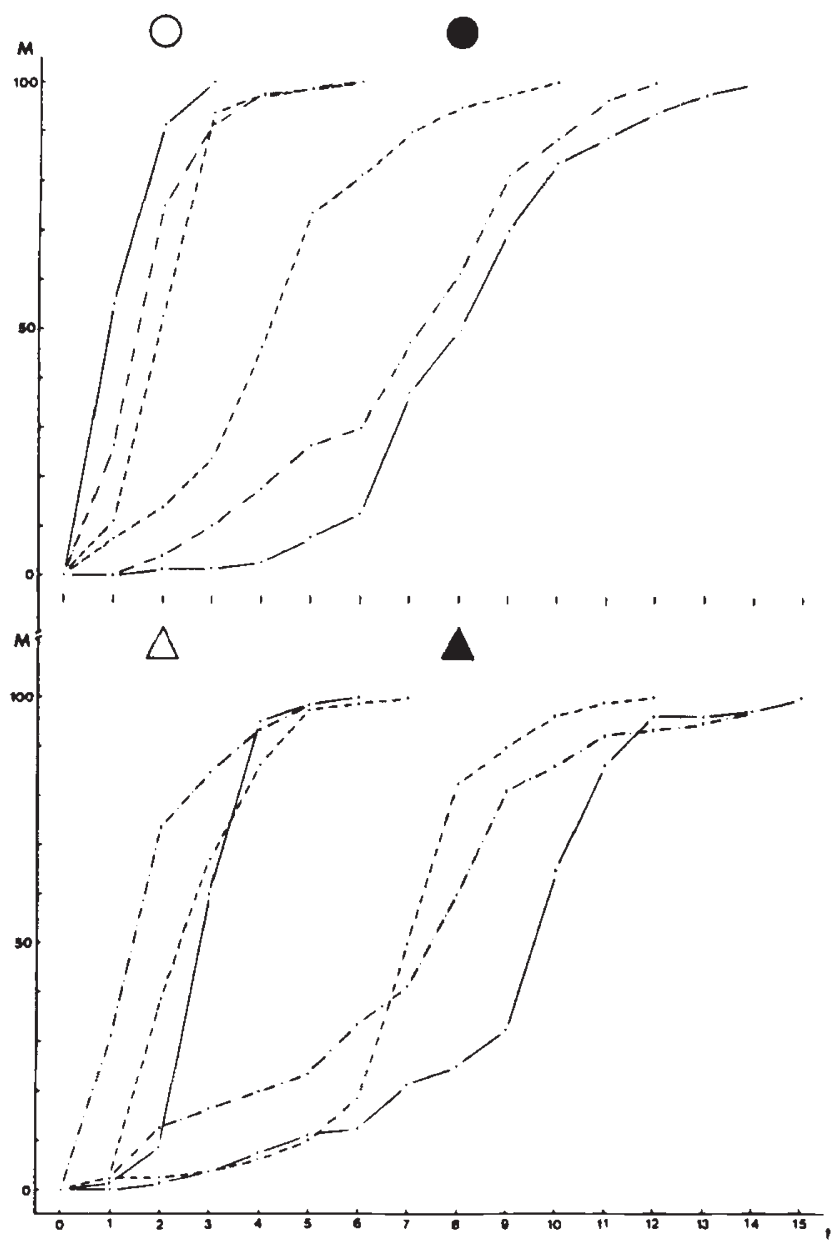

FIG. 3. Adult mortality $(\mathbf{M})$ curves of $\operatorname{LRC}(O, O)$ and $\operatorname{LRSe}(\Delta, \Delta)$ lines on basic $(O, \Delta)$ or a 11 per cent ethanol basic medium $(\mathbf{O}, \Delta)$, as a function of the number of days $(t)$.

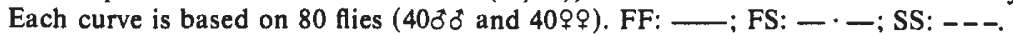

alcoholic medium), and a higher resistance to starvation (to 1.89 days at FF) and a more efficient ethanol utilisation (to an increase of 2.86 days in the life duration for SS) for both homozygotes. For both ethanol tolerance and developmental times, the positive effects of selection are higher in the supplemented medium (see also fig. 2 ), which can explain the line $\times$ medium interaction (see ANOVA in table 4). As life span data from non-alcoholic and alcoholic media have different significance, the effects of the selection do not have to be related. However, both the effects of resistance to starvation and of alcohol utilisation are improved by the selection (see also fig. 3) only in the homozygotes. As the heterozygotes seems not to be improved for this fitness component, different systems (probably involving Adh locus) for increasing life span may have been selected at each homozygous line. Further experiments must be done. 
G. DORADO AND M. BARBANCHO

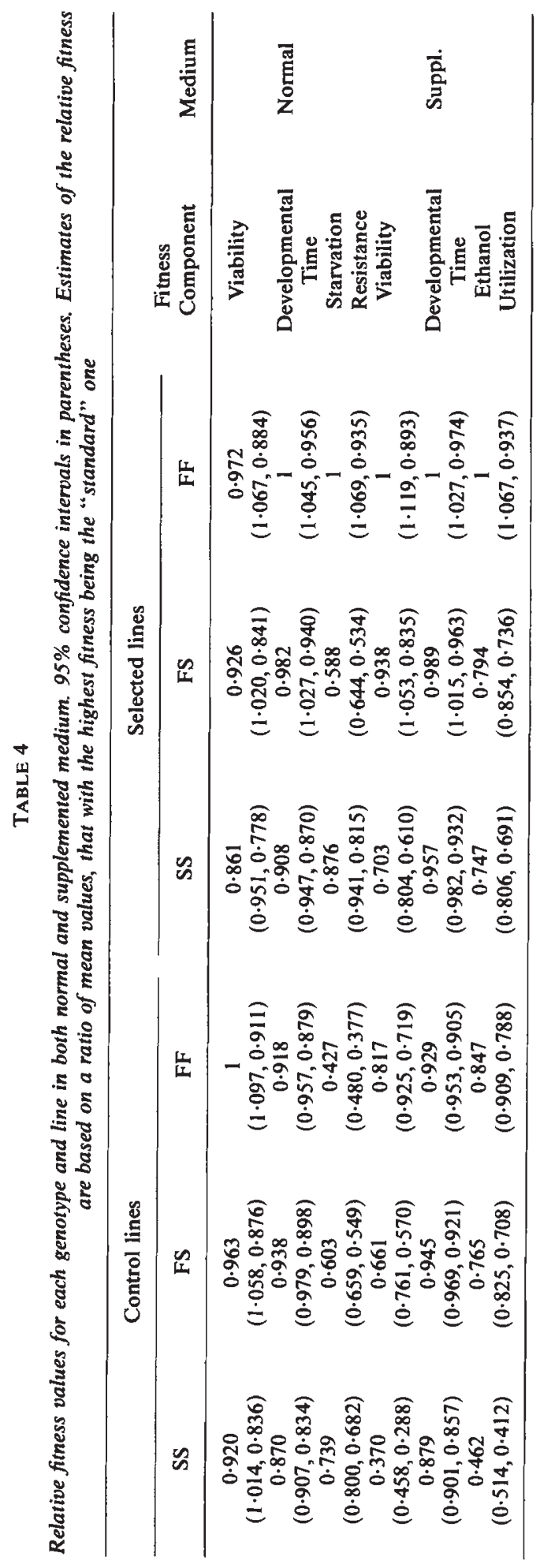




\section{(v) Relative fitnesses}

To summarise the results, relative fitnesses for each genotype, fitness component and medium are shown in table 4 . To estimate the relative fitnesses for each component, the ratio of each mean value to the one with the highest fitness within each medium was used. To estimate the error variances of the standard values for each medium, the respective error mean squares of the analyses of variances were used (see Bliss, 1967).

In general, for all three components studied and in both regular and supplemented medium, the FF homozygotes at the selected lines show the highest relative fitness, this superiority being clearer in the alcoholic media than in non-alcoholic ones.

\section{Discussion}

First of all, we must say that it is unreasonable to attribute all the changes only to selection at the $A d h$ locus. Linked unknown genes or even independent genes could be involved. However, our results, in general, are in agreement with others taken from widely separated populations (for example, the Colmar strain, France (David and Bocquet, 1977); Kaduna, Nigeria (Daly and Clarke, 1981); Groningen, The Netherlands (van Delden et al., 1978; van Delden and Kamping, 1979); Puerto Asis, Columbia (van Herewege and David, 1980)).

Van Delden and Kamping (1979), studying the developmental times of Groningen strains for Adh locus on regular medium, asked themselves if the shorter developmental time of FF and FS larvae was a general phenomenon. We have found, on regular food, slower developmental times of SS homozygotes and, moreover, the developmental times for all three genotypes have been significantly decreased by the alcohol selection. Colmar selected lines also show a faster developmental time than non-selected ones, though the alcohol selection had eliminated $A d h^{\text {s }}$ alleles (David and Bocquet, 1977). As these differences in developmental times are found in non-alcoholic media, another role must be postulated for the $\mathrm{ADH}$ isozymes in addition to the detoxification of alcohols if the Adh locus is involved in developmental time. On the other hand, the ethanol in the medium produces a considerable delay in developmental time, though this delay is lower in the selected lines. Oakeshott (1976) linearly relates the differences in developmental time in the ethanol supplemented medium to the differences in the ADH activity. However, doubts about the relationships between the ADH activity alone and developmental time came from the same author (Oakeshott, 1977) on using the pentenol supplemented medium. Selective differences in developmental time were similar in direction for both ethanol and pentenol although Sofer and Hatkoff (1972) suggested that, in vivo, ADH oxidizes pentenol into a lethal ketone, ethyl vinyl ketone. Our results increase these doubts, as SS homozygotes seem to have the highest relative reduction in developmental time.

In relation to egg-to-adult viability, in general, no significant intergenotypic differences were observed in normal medium for this fitness component. However, on supplemented medium, significant differences can be noted, homozygous SS being less tolerant, from the first generation of selection (control lines at 11 per cent ethanol medium), which supports the 
notion that $\mathrm{ADH}$ isozymes must play an important role in ethanol detoxification. Although there are exceptions (Gibson et al., 1979; Oakeshott, 1979; Ziolo and Parsons, 1982), all from Australian winery populations, most of the papers suggest that alcohol treatments modify $A d h$ allele frequencies (Gibson, 1970; Bijlsma-Meeles and van Delden, 1974; van Delden et al., 1975, 1978; Cavener and Clegg, 1978, 1981) by favouring the most active one.

Our results show that the selected flies having the $A d h^{\mathrm{F}}$ allele are more tolerant than homozygous SS ones which is in agreement with the above results. This dominance of the $A d h^{\mathrm{F}}$ allele over the $A d h^{\mathrm{S}}$ one was also observed by Briscoe et al. (1975), from a Spanish winery population. However, homozygous SS flies are the most improved by selection for ethanol tolerance. Several papers support the idea that ADH plays a significant role in the detoxification of ethanol (David et al., 1976, 1978, 1981; Kamping and van Delden, 1978) and of isopropanol and acetone (David et al., 1981). However, ethanol detoxification and ethanol utilisation as food, both depending on an active $\mathrm{ADH}$, seem to be controlled by different genetic mechanisms (van Herrewege and David, 1980; David et al., 1981). General ethanol detoxification processes involve its conversion into acetate, by acetaldehyde, which is further transformed into metabolic energy in the Krebs cycle (Clarke, 1975; David et al., 1976; DeltombeLietaert et al., 1979).

Our results clearly support the view that ethanol can be used as food by Drosophila melanogaster. For both control and selected lines and all three genotypes, ethanol considerably increases the survival of adults. In addition, selection has improved ethanol utilisation, although only for FF and SS homozygotes. Again, the homozygous SS line has been the most improved, but FF flies are always the best at using ethanol as food. Similar results were obtained for both, high (Briscoe et al., 1975; Morgan, 1975; Libion-Mannaert et al., 1976) and low ethanol concentrations (Daly and Clarke, 1981). That the better ability to use ethanol for FF flies is not due to a higher toxicity for the SS ones is suggested by a much better life span increase of the SS flies on alcohol than any of the other flies on water alone.

On the other hand, ethanol tolerance and ethanol utilisation do not seem to be related. While selection has decreased the egg-adult mortality for all three genotypes, ethanol utilisation has only been improved for both homozygotes. This fact seems to support the hypothesis of partial independence of these components, proposed by van Herrewege and David (1980).

Finally, we can point out the changes in the resistance to starvation brought about by the ethanol selection. These changes seem not to be related to any known role of the Adh locus. However such changes have also been reported in other papers (David and Bocquet, 1977; van Herewege and David, 1980), associated with alcohol selection. Further experiments would be interesting.

In conclusion, the ethanol selection has resulted successfully for all three fitness components studied. After selection a higher ethanol tolerance, a better ability to use ethanol as food and a shorter developmental time have been observed when flies are grown on ethanol supplemented media. Though SS homozygotes have been the most improved, FF homozygotes have the highest relative fitness for all three components. This highest fitness of the FF homozygotes is in agreement with the rapid increase of the $A d h^{\mathrm{F}}$ allele when polymorphic populations for the $A d h$ locus are submitted to 
an ethanol selection. Without doubt, the high ethanol tolerance of $D$. melanogaster has certainly a great ecological significance (McKenzie and Parsons, 1974) and such a trait must involve a complex genetic system, in which the Adh locus plays an important role. When a population with enough genetic variability is submitted to an ethanol stress, any genetic change providing a better and faster adaptive response (a $A d h^{\mathrm{F}}$ allele, for example) can be favourably selected though sometimes a specific physiological change can not be associated with a given genetic one.

\section{REFERENCES}

BIJLSMA-MEELES, E. AND VAN DELDEN, w. 1974. Intra- and interpopulation selection concerning the alcohol dehydrogenase locus in Drosophila melanogaster. Nature, 247, 369-371. BLISS, C. I. 1967. Statistics in Biology (Vol. 1). McGraw-Hill. U.S.A.

BRISCOE, D. A., ROBERTSON, A. AND MALPICA, J. M. 1975. Dominance at Adh locus in response of adult Drosophila melanogaster to environmental alcohol. Nature, 255, 148-149.

CAVENER, D. R. AND CLEGG, M. T. 1978. Dynamics of correlated genetic systems. IV. Multilocus effects of ethanol stress environments. Genetics, 90, 629-644.

CAVENER, D. R. AND CLEGG, M. T. 1981. Multigenic response to ethanol in Drosophila melanogaster. Evolution, 35, 1-10.

CLARKE, B. 1975. The contribution of ecological genetics to evolutionary theory: detecting the direct effects of selection on particular polymorphic loci. Genetics, 79, 101-113.

DALY, K. AND CLARKE, B. 1981. Selection associated with the alcohol dehydrogenase locus in Drosophila melanogaster: differential survival of adults maintained on low concentrations of ethanol. Heredity, 46, 219-226.

DAVID, J. R. AND BOCQUET, C. 1977. Genetic tolerance to ethanol in Drosophila melanogaster: increase by selection and analysis of correlated responses. Genetica, 47, 43-48.

DAVID, J. R., BOCQUET, C., ARENS, M. F. AND FOUILLET, P. 1976. Biological role of alcohol dehydrogenase in the tolerance of Drosophila melanogaster to aliphatic alcohols: utilization of an ADH-null mutant. Biochem. Genet., 14, 989-997.

DAVID, J. R., BOCQUET, C., VAN HERREWEGE, J., FOUILLET, P. AND ARENS, M. F. 1978. Alcohol metabolism in Drosophila melanogaster: uselessness of the most active aldehyde oxidase produced by the Aldox locus. Biochem. Genet., 16, 203-211.

DAVID, J. R., VAN HERREWEGE, J., SCHEEMAEKER-LOUIS, M. DE AND PLA, E. 1981. Drosophila alcohol dehydrogenase: detoxification of isopropanol and acetone, substances not used in energy metabolism. Heredity, 47, 263-268.

DELTOMBE-LIETAERT, M. C., DelCouR, J., LeNELle-MONFORT, N. AND EleNS, A. 1979. Ethanol metabolism in Drosophila melanogaster. Experientia, 35, 579-581.

GIBSON, J. 1970. Enzyme flexibility in Drosophila melanogaster. Nature, 227, 959-960.

GIBSON, J. B., LEWIS, N., ADENA, M. A. AND WILSON, S. R. 1979. Selection for ethanol tolerance in two populations of Drosophila melanogaster segregating alcohol dehydrogenase allozymes. Aust. J. Biol. Sci., 32, 387-398.

GIBSON, J. B. AND MIKLOVICH, R. 1971. Modes of variation in alcohol dehydrogenase in Drosophila melanogaster. Experientia, 27, 99-100.

KAMPING, A. AND VAN DELDEN, w. 1978. Alcohol dehydrogenase polymorphism in populations of Drosophila melanogaster. II. Relation between ADH activity and adult mortality. Biochem. Genet., 16, 541-551.

LEWONTIN, R. C. 1974. The Genetic Basis of Evolutionary Change. Columbia University Press, New York.

LIBION-MANNAERT, M., DELCOUR, J., DELTOMBE-LIETAERT, M. M., LENELLE-MONTFORT, N. AND ELENS, A. 1976. Ethanol as a "food" for Drosophila melanogaster: influence of the ebony gene. Experientia, 32, 22-24.

MCKECHNIE, S. M. AND MORGAN, P. 1982. Alcohol dehydrogenase polymorphism of Drosophila melanogaster: aspects of alcohol and temperature variation in the larval environment. Aust. J. Biol. Sci., 35, 85-93.

MCKENZIE, J. A. AND PARSONS, P. A. 1974. Microdifferentiation in a natural population of Drosophila melanogaster to alcohol in the environment. Genetics, 77, 385-394.

MORGAN, P. 1975. Selection acting directly on an enzyme polymorphism. Heredity, 35, 124-127. 
OAKESHOTT, J. G. 1976. Selection at the alcohol dehydrogenase locus in Drosophila melanogaster imposed by environmental ethanol. Genet. Res., 26, 365-373.

OAKESHOTT, J. G. 1977. Variation in the direction of selection applied by pentenol to the alcohol dehydrogenase locus in Drosophila melanogaster. Aust. J. Biol. Sci., 30, 259-267.

OAKESHOTT, J. G. 1979. Selection affecting enzyme polymorphisms in laboratory populations of Drosophila melanogaster. Oecologia, 143, 341-354.

SOFER, W. H. AND HATKOFF, M. A. 1972. Chemical selection of alcohol dehydrogenase negative mutants in Drosophila. Genetics, 72, 545-549.

THOMPSON, J. N. JR. AND KAISER, T. N. 1977. Selection acting upon slow-migrating $A d h$ alleles differing in enzyme activity. Heredity, 38, 191-195.

VAN DELDEN, W., BOEREMA, A. C. AND KAMPING, A. 1978. The alcohol dehydrogenase polymorphism in populations of Drosophila melanogaster. I. Selection in different environments. Genetics, 90, 161-191.

VAN DELDEN, W. AND KAMPING, A. 1979. The alcohol dehydrogenase polymorphism in populations of Drosophila melanogaster. III. Differences in developmental times. Genet. Res. Camb., 33, 15-27.

VAN DELDEN, W., KAMPING, A. AND VAN DIJK, H. 1975. Selection at the alcohol dehydrogenase locus in Drosophila melanogaster. Experientia, 31, 418-420.

VAN HERREWEGE, J. AND DAVID, J. R. 1980. Alcohol tolerance and alcohol utilization in Drosophila: partial independence of two adaptive traits. Heredity, 44, 229-235.

WILSON, S. R., OAKESHOTT, J. G., GIBSON, J. B. AND ANDERSON, P. R. 1982. Measuring selection coefficients affecting the alcohol dehydrogenase polymorphism in Drosophila melanogaster. Genetics, 100, 113-126.

ZIOLO, L. K. AND PARSONS, P. A. 1982. Ethanol tolerance, alcohol-dehydrogenase activity and Adh allozymes in Drosophila melanogaster. Genetica, 57, 231-237. 